\title{
Interleukin-6 signaling in patients with chronic heart failure treated with cardiac resynchronization therapy
}

\author{
Katarzyna Ptaszynska-Kopczynska ${ }^{1}$, Anna Szpakowicz ${ }^{1}$, Marta Marcinkiewicz-Siemion ${ }^{1}$, \\ Anna Lisowska ${ }^{1}$, Ewa Waszkiewicz ${ }^{1}$, Marcin Witkowski ${ }^{1}$, Piotr Jakim², Bogdan Galar², \\ Wlodzimierz J. Musial ${ }^{1}$, Karol A. Kamiński ${ }^{1,3}$
}

\begin{abstract}
'Department of Cardiology, Medical University of Bialystok, Bialystok, Poland ${ }^{2}$ Department of Cardiology, Internal Affair and Administration Ministry Hospital, Bialystok, Poland

${ }^{3}$ Department of Community Medicine and Civilization Disease Prevention, Medical University of Bialystok, Bialystok, Poland
\end{abstract}

Submitted: 9 October 2015

Accepted: 28 December 2015

Arch Med Sci 2017; 13, 5: 1069-1077

DOI: 10.5114 /aoms.2016.58635

Copyright @ 2016 Termedia \& Banach

\begin{abstract}
Introduction: Increased expression of interleukin-6 (IL-6) has been described in left ventricular dysfunction in the course of chronic heart failure. Cardiac resynchronization therapy (CRT) is a unique treatment method that may reverse the course of chronic heart failure (CHF) with reduced ejection fraction (HF-REF). We aimed to evaluate the IL-6 system, including soluble IL-6 receptor (sIL-6R) and soluble glycoprotein 130 (sgp130), in HF-REF patients, with particular emphasis on CRT effects.

Material and methods: The study enrolled 88 stable HF-REF patients (63.6 \pm 11.1 years, 12 females, $E F<35 \%$ ) and 35 comorbidity-matched controls (63.5 \pm 9.8 years, 7 females). Forty-five HF-REF patients underwent CRT device implantation and were followed up after 6 months. Serum concentrations of IL-6, sIL-6R and sgp130 were determined using ELISA kits.

Results: The HF-REF patients had higher IL-6 (median: 2.6, IQR: 1.6-3.8 vs. 2.1, IQR: $1.4-3.1 \mathrm{pg} / \mathrm{ml}, p=0.03$ ) and lower sIL-6R concentrations compared to controls (median: 51, IQR: $36-64$ vs. 53. IQR $44-76 \mathrm{ng} / \mathrm{ml}, p=0.008$ ). There was no significant difference between sgp130 concentrations. In the HF-REF group IL-6 correlated negatively with EF $(r=-0.5, p=0.001)$ and positively with BNP $(r=0.5, p=0.008)$ and CRP concentrations $(r=0.4, p=$ 0.02 ). Patients who presented a positive response after CRT showed a smaller change of sIL-6R concentration compared to nonresponders ( $\Delta$ sIL-6R: -0.2 \pm 7.1 vs. $7 \pm 14 \mathrm{ng} / \mathrm{ml} ; p=0.04$ ).

Conclusions: HF-REF patients present higher IL- 6 and lower sIL-6R levels. IL-6 concentration reflects their clinical status. CRT-related improvement of patients' functional status is associated with a smaller change of sIL-6R concentration in time.
\end{abstract}

Key words: cardiac resynchronization therapy, heart failure, interleukin-6, soluble glycoprotein 130 , IL-6 receptor.

\section{Introduction}

Chronic heart failure (CHF) is one of the most common cardiovascular disorders. It is associated with unfavorable outcome and often coexists with other chronic diseases that further affect the prognosis [1, 2]. Among a variety of parameters reflecting disease severity, left ventricular

\section{Corresponding author:} Prof. Karol A. Kamiński MD, PhD Department of Cardiology Medical University of Bialystok 24 a Sklodowskiej St 15-276 Bialystok, Poland Phone: +48 857468656 Fax: +48 857468604 E-mail: fizklin@wp.pl 
ejection fraction (LVEF) remains one of the strongest predictors of long-term prognosis in those patients. In recent years a significant improvement of CHF patients' therapy has been observed. One of the treatment methods dedicated for patients with heart failure with reduced ejection fraction (HF-REF) and ventricular conduction disturbances is cardiac resynchronization therapy (CRT) [3]. Cardiac resynchronization therapy effectiveness is based on biventricular pacing, which can improve left ventricular systolic function by correcting the electromechanical dyssynchrony [4]. This results in an improvement of patients' functional capacity as well as reduction of rehospitalizations and mortality. However, every third patient does not benefit from the therapy $[5,6]$. Therefore, the mechanisms as well as novel diagnostic methods predicting the unsatisfactory CRT response are sought $[7,8]$.

Inflammatory activation is involved in the pathogenesis of many cardiovascular disorders, including left ventricular dysfunction. Both experimental and clinical evidence suggests that proinflammatory cytokines play an important role in progression and unfavorable outcome of the disease. Interleukin 6 (IL-6) is one of the most important mediators in cardiovascular disorders and is considered a biomarker as well as a potential target for the therapy [9]. However, its role in both cardiovascular physiology and pathology is often unclear and heavily disputed $[9,10]$. Interleukin 6 signals through the transmembrane receptor composed of a specific IL-6R and catalytic subunit gp130 (glycoprotein 130), while the soluble receptors IL-6 receptor (sIL-6R) and glycoprotein 130 (sgp130) act as additional IL-6 activity regulators. Soluble IL-6R after formation with available IL- 6 of the IL-6/sIL-6R complex is capable of binding to membrane glycoprotein 130 (gp130) and inducing intracellular signaling. This phenomenon, called trans-signaling, enables IL- 6 to stimulate the cells that lack an endogenous membrane-bound IL- 6 receptor (mIL-6R). The regulating mechanism of production of sgp130 as well as sIL6-R is still unclear. Both of them are produced by proteolytic cleavage and shedding of the membrane-anchored receptors and by alternative mRNA splicing [11]. Given the controversies whether cardiomyocytes have membrane-bound IL-6R, IL-6 activity in the heart is very likely to be based on trans-signaling. The activity of the sIL-6R/IL-6 complex is naturally balanced by the presence of a soluble form of gp130 (sgp130), which is a decoy receptor, functioning as an inhibitor of IL-6/sIL-6R complex activity and is a crucial inhibitory regulator of the IL- 6 pathway in human heart $[12,13]$.

High levels of IL- 6 and sIL-6R have been reported in several chronic inflammatory and autoimmune diseases as well as in cardiovascular disorders [14, 15]. Investigators have demonstrated elevated circulating levels of IL-6 and sIL-6R in HF patients, with a progressive increase in relation to neurohormonal activation as well as decreasing functional capacity and left ventricle (LV) function [9, 16]. Moreover, elevated myocardial gene expression of this cytokine was observed in end-stage human HF of both ischemic and non-ischemic etiology $[14,17]$. Interestingly, it was suggested that IL-6-related sustainable gp130 signaling may be cardioprotective, whereas excessive activation of gp130 results in maladaptation and HF [18].

In this study we aimed to evaluate and compare three components of IL- 6 signaling in $\mathrm{CHF}$ patients. Therefore we studied the association between serum IL-6 and its soluble receptors sgp130 and sIL-6R in the context of clinical as well as biochemical evidence of LV dysfunction. Another aspect is to look at CRT as a unique CHF treatment to get a better insight into mechanisms underlying the reversal of the disease course.

\section{Material and methods}

The study included 88 patients with stable, optimally pharmacologically treated chronic heart failure with ejection fraction lower than 35\% assessed in echocardiography (HF-REF group) (Table I).

The CHF etiology included ischemic (51 (58\%) patients) and dilated (37 (42\%) patients) cardiomyopathy. Forty-five of these patients with QRS complex duration $>120$ ms and left bundle branch block (LBBB) (41 patients) or > 150 ms with nonLBBB QRS morphology (4 patients) further underwent the procedure of CRT device implantation regardless of the leading rhythm (sinus or atrial fibrillation). Implanted CRT devices were produced by Medtronic (75\% of devices) and Biotronik (25\%). More than $97 \%$ of biventricular pacing was considered as satisfactory for Medtronic or fulfilling the advanced resynchronization criteria for the Biotronik device $[19,20]$. The percentage of biventricular pacing was specified on the basis of simultaneous stimulation of both left and right ventricles detected by the CRT device.

The reference cohort consisted of 35 volunteers (outpatient) matched for age, sex, body weight (Table I) and comorbidities such as hypertension, diabetes mellitus, ischemic heart disease or atrial fibrillation. All patients underwent the same diagnostic assessment: a medical history with assessment of NYHA functional class, physical examination, transthoracic echocardiography, cardiopulmonary exercise test (CPET), 6-minute walk test (6MWT) and venous blood tests. Forty-five HF-REF patients treated with CRT underwent this same procedure after 6 months. Patients with active or recent infection or moderate to 
Table I. Demographic, functional, echocardiographic and laboratory characteristics of chronic heart failure patients and control group

\begin{tabular}{|c|c|c|c|}
\hline Parameter & HF-REF & Control & $P$-value \\
\hline Patients, $n$ & 88 & 35 & \\
\hline Age [years] & $63.6 \pm 11.1$ & $63.5 \pm 9.8$ & 0.9 \\
\hline Female gender, $n(\%)$ & $12(13.6)$ & $7(20)$ & 0.4 \\
\hline $\mathrm{BMI}\left[\mathrm{kg} / \mathrm{m}^{2}\right]$ & $28.7 \pm 4.4$ & $28.3 \pm 4$ & 0.6 \\
\hline \multicolumn{4}{|l|}{ Pharmacotherapy: } \\
\hline Angiotensin-converting-enzyme inhibitor, $n(\%)$ & $74(84)$ & $12(34)$ & $<0.001$ \\
\hline Angiotensin receptor blocker, $n(\%)$ & $10(13)$ & $6(17)$ & 0.2 \\
\hline$\beta$-blocker, $n(\%)$ & $86(98)$ & $18(51)$ & $<0.001$ \\
\hline Aldosterone antagonist, $n(\%)$ & $82(93)$ & $5(14)$ & $<0.001$ \\
\hline Loop diuretic, $n(\%)$ & $75(85)$ & $5(14)$ & $<0.001$ \\
\hline Statin, $n(\%)$ & $71(81)$ & $11(31)$ & $<0.001$ \\
\hline Acetylsalicylic acid, $n(\%)$ & $56(64)$ & $10(29)$ & $<0.001$ \\
\hline \multicolumn{4}{|l|}{ Functional parameters: } \\
\hline NYHA functional class & $2.6 \pm 0.6$ & - & \\
\hline 6MWD [m] & $377.4 \pm 120$ & $518.3 \pm 98.7$ & $<0.001$ \\
\hline Peak $\mathrm{VO}_{2}[\mathrm{ml} / \mathrm{kg} / \mathrm{min}]$ & $17.1 \pm 6.61$ & $23.8 \pm 5.9$ & $<0.001$ \\
\hline Peak $\mathrm{VO}_{2}(\%$ of predicted $)$ & $60.1 \pm 21.3$ & $85.7 \pm 29.2$ & $<0.001$ \\
\hline Peak VD/VT & $0.2 \pm 0.07$ & $0.17 \pm 0.055$ & 0.04 \\
\hline VE/VCO 2 slope & $32.8 \pm 8.2$ & $27.3 \pm 3.6$ & $<0.001$ \\
\hline \multicolumn{4}{|l|}{ Echocardiography: } \\
\hline $\mathrm{EF}(\%)$ & $24.3 \pm 6.8$ & $62 \pm 6.1$ & $<0.001$ \\
\hline LVEDV [ml] & $224 \pm 81.7$ & $96.8 \pm 24.3$ & $<0.001$ \\
\hline LVESV [ml] & $173.8 \pm 72.1$ & $37.7 \pm 12$ & $<0.001$ \\
\hline \multicolumn{4}{|l|}{ Laboratory analyses: } \\
\hline BNP [pg/ml] & 190.9, IQR 88-411 & 25.7, IQR 14.7-48.1 & $<0.001$ \\
\hline $\mathrm{CRP}[\mathrm{mg} / \mathrm{dl}]$ & 1.9, IQR $1.15-3.1$ & 1.5, IQR 0.9-2.4 & 0.2 \\
\hline Erythrocytes $\left[10^{6} / \mu \mathrm{l}\right]$ & $4.6 \pm 0.5$ & $4.9 \pm 0.5$ & 0.003 \\
\hline Hemoglobin [g/dl] & $13.9 \pm 1.3$ & $14.4 \pm 1.8$ & 0.005 \\
\hline Creatinine $[\mathrm{mg} / \mathrm{dl}]$ & $1.1 \pm 0.3$ & $0.9 \pm 0.2$ & 0.01 \\
\hline Uric acid [mg/dl] & $7.1 \pm 1.9$ & $6 \pm 1.6$ & 0.03 \\
\hline \multicolumn{4}{|l|}{ IL-6 signaling: } \\
\hline Interleukin-6 [pg/ml] & 2.6, IQR 1.6-3.8 & 2.1, IQR 1.4-3.1 & 0.03 \\
\hline Soluble IL-6 receptor [ng/ml] & $51.7 \pm 18.9$ & $63.3 \pm 27.6$ & 0.008 \\
\hline Soluble glycoprotein 130 [ng/ml] & $310.9 \pm 68.2$ & $306.5 \pm 59.4$ & 0.7 \\
\hline
\end{tabular}

severe chronic lung disease were excluded from the study. Two-dimensional echocardiographic measurements included analysis with Simpson's method. Echocardiography was also used to exclude clinically silent but significant heart abnormalities in the control group. A positive response to CRT was defined by improvement in clinical CHF symptoms (lower NYHA class, longer 6MWT distance) and at least a $15 \%$ relative reduction in
LV end-systolic volume (LVESV) in echocardiography, indicating reverse remodeling, according to previous studies [21, 22].

Cardiopulmonary exercise test was performed using symptoms limited treadmill exercise with the RAMP protocol in both groups. Of the numerous parameters, peak oxygen uptake (peak $\mathrm{VO}_{2}$ and percentage (\%) of predicted), dead space/tidal volume (VD/VT) ratio and the slope of the VE/ 
$\mathrm{VCO}_{2}$ relationship from the initiation to peak exercise (VE/VCO 2 slope) were used for the analysis.

Fasting peripheral venous blood samples were obtained from HF-REF patients and controls. Serum aliquots of about $1.5 \mathrm{ml}$ were stored at $-80^{\circ} \mathrm{C}$ for future analysis. Concentrations of IL-6, sIL-6R and sgp130 were determined using commercially available ELISA kits (R\&D Systems, USA), according to the manufacturer's instructions. The mean minimum detection limit was $0.04 \mathrm{pg} / \mathrm{ml}$ for IL-6 High Sensitivity, 6.5 pg/ml for IL-6sR and $0.08 \mathrm{ng} /$ $\mathrm{ml}$ for sgp130. ELISA assays recognized both free cytokines and their complexes. Blood samples were also analyzed for B-type natriuretic peptide (BNP), C-reactive protein (CRP), blood count, lipid profile, uric acid and creatinine concentrations in the local laboratory.

The study was approved by the institutional medical ethics committee and complied with the Declaration of Helsinki. All patients were included in the study after signing informed consent for participating in the study, including taking and storage of blood samples.

\section{Statistical analysis}

The distribution of all variables was verified with the Kolmogorov-Smirnov test. Data are expressed as mean \pm standard deviation (SD) or median values with interquartile range (IQR) as appropriate. Statistical analysis was performed using Student's t-test or Mann-Whitney $U$ test for continuous data depending on distribution. Comparisons of variables between baseline and

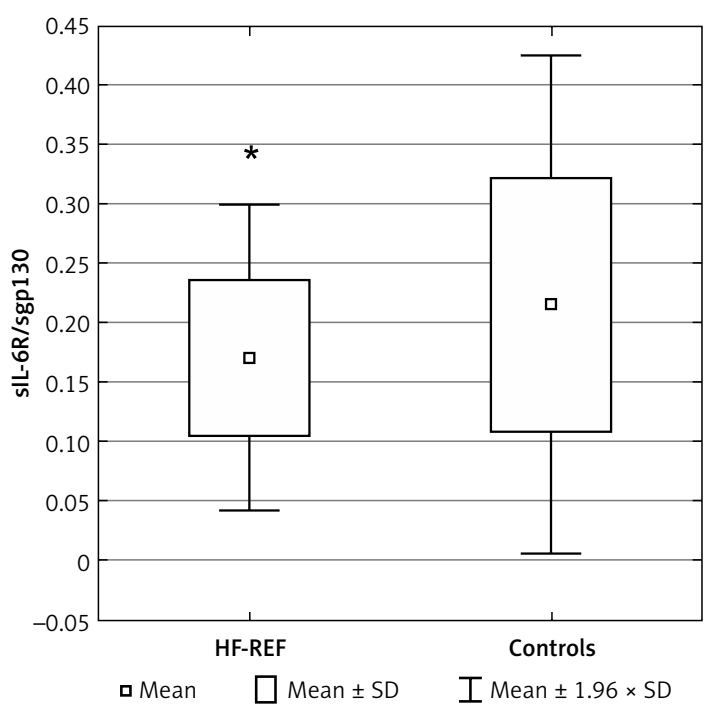

Figure 1 . The ratio of serum concentrations of soluble interleukin-6 receptor (sIL-6R) and soluble glycoprotein 130 (sgp130) in chronic heart failure with reduced ejection fraction (HF-REF) patients $(0.17 \pm 0.07)$ and controls (0.22 \pm 0.11$)$ ${ }^{\star} p=0.006$. follow-up values were performed using paired Student's $t$-test or Wilcoxon's test. Where appropriate, Pearson's or Spearman's correlation coefficients were used to examine the relationship between two continuous variables. $P$-value $<0.05$ was considered as statistically significant. The statistical software package Statistica 10 (StatSoft, USA) was used for analysis.

\section{Results}

The clinical and biochemical characteristics of the HF-REF and control group are shown in Table I. HF-REF patients had higher IL-6 and lower sIL-6R serum concentrations compared to controls, while there was no significant difference between sgp130 concentrations (Table I). Furthermore, the analysis of SIL-6R to sgp130 ratio showed lower values in HF-REF compared to the control group (0.17 \pm 0.07 vs. $0.22 \pm 0.11, p=0.006)$ (Figure 1).

In the HF-REF group the baseline concentrations of sgp130 correlated with IL- $6(r=0.4$, $p=0.001)$ and SIL-6R $(r=0.2, p=0.02)$. Moreover, IL-6 concentration correlated positively with BNP $(r=0.5, p=0.008)$ and CRP concentrations $(r=0.4, p=0.02)$. Concentration of sgp130 correlated with $\operatorname{BNP}(r=0.4, p<0.001)$.

In HF-REF patients IL-6 level correlated negatively with left ventricular $\operatorname{EF}(r=-0.5, p=0.001)$, while sIL-6R correlated negatively with LVEDV $(r=-0.3, p=0.03)$. Among CPET parameters dead space/tidal volume (VD/VT) ratio and $\mathrm{VE} / \mathrm{VCO}_{2}$ slope correlated with sIL-6R level $(r=0.3, p=0.03$; $r=0.3, p=0.04$, respectively).

After 6 months of CRT significant improvement in NYHA functional class $(2.3 \pm 0.4$ vs. $2.8 \pm 0.4$, $p<0.001), 6$ MWD (417 \pm 98.9 vs. $367.3 \pm 108.1 \mathrm{~m}$, $p<0.001)$ and echocardiographic parameters, e.g. EF $(32.5 \pm 9.5 \%$ vs. $23.6 \pm 6.1 \%, p<0.001)$ and LVESV (141.7 \pm 83.8 vs. $189.5 \pm 67.6 \mathrm{ml}, p=0.014)$, were observed (Table II). During this observational period no significant changes in pharmacotherapy were made.

There were no significant differences in baseline concentrations of $\mathrm{IL}-6$ and the receptors sIL-6R and sgp130 between groups of HF-REF patients who were considered to be responders $(n=30)$ or nonresponders $(n=15)$ according to the clinical response to CRT (Table III).

After 6 months, 30 patients who benefited from resynchronization (responders) showed a smaller change of sIL-6R concentration when compared to those with worse outcome (nonresponders) ( $\Delta$ sIL-6R: $-0.2 \pm 7.1$ vs. $7 \pm 14 \mathrm{ng} / \mathrm{ml} ; p=0.04$ ) (Figure 2).

Such changes of IL- 6 and sgp130 were not observed (Figures 3 and 4).

After 6 months of CRT, IL- 6 concentration correlated negatively with $6 \mathrm{MWT}$ distance $(r=-0.4$, 
$p=0.01)$ and positively with VD/VT $\max (r=0.4$ $p=0.02$ ).

The analysis of the CRT group divided according to BNP concentration into patients with normal (BNP $\leq 100 \mathrm{pg} / \mathrm{ml}$ ) and increased concentration revealed no differences at baseline, while after 6 months of CRT patients with elevated BNP had higher concentrations of IL-6 (3.3, IQR: 1.9-4.4 vs. 1.7, IQR: $1.2-2.1 \mathrm{pg} / \mathrm{ml}, p=0.02)$ and sgp130 (328 \pm 71.7 vs. $278 \pm 64.6 \mathrm{ng} / \mathrm{ml}, p=0.03$ ).

The analysis of percentage of resynchronization revealed no significant differences between responders and nonresponders (99.7 $\pm 0.75 \%$ vs. $97.6 \pm 5.2 \%$, respectively $p=0.116$ ). An unsatisfactory percentage of resynchronization in 6-month observation was observed in 6 (13.3\%) patients, all of them nonresponders according to combined clinical and echocardiographic criteria, while in the responders group all patients presented a good percentage of biventricular pacing $(p=0.002)$. Hence, a good percentage of resynchronization did not ensure a positive response to CRT, but an unsatisfactory percentage of biventricular pacing led to a lack of clinical benefit from CRT.

\section{Discussion}

In the present study we analyzed the association of IL- 6 and its two soluble receptors sgp 130 and IL-6R with the clinical course of patients with chronic heart failure with reduced ejection fraction. The analysis of the HF-REF group revealed higher IL-6 and lower sIL-6R serum levels when compared to controls, while sgp130 concentra-

Table II. Characteristics of chronic heart failure patients treated with cardiac resynchronization therapy at baseline and 6-month follow-up

\begin{tabular}{|c|c|c|c|c|c|c|}
\hline Parameter & $\begin{array}{l}\text { CRT group } \\
\text { baseline }\end{array}$ & $\begin{array}{l}\text { CRT group } \\
6 \mathrm{M}\end{array}$ & $P$-value & $\begin{array}{c}\text { Nonresponders } \\
6 \mathrm{M} \\
\end{array}$ & $\begin{array}{c}\text { Responders } \\
6 \mathrm{M}\end{array}$ & $P$-value \\
\hline Patients, $n$ & 45 & 45 & & 15 & 30 & \\
\hline Age [years] & $64.6 \pm 10.4$ & $65 \pm 10.6$ & & $65.9 \pm 10.2$ & $64.5 \pm 10.8$ & 0.5 \\
\hline Female gender, $n(\%)$ & $7(24.4)$ & $7(24.4)$ & & $1(7.1)$ & $6(19.3)$ & 0.2 \\
\hline BMI $\left[\mathrm{kg} / \mathrm{m}^{2}\right]$ & $28.5 \pm 4$ & $28.9 \pm 4.1$ & 0.06 & $29.2 \pm 4.8$ & $28.2 \pm 3.7$ & 0.4 \\
\hline \multicolumn{7}{|l|}{ Functional parameters: } \\
\hline NYHA functional class & $2.8 \pm 0.4$ & $2.3 \pm 0.4$ & $<0.001$ & $2.4 \pm 0.4$ & $2.3 \pm 0.5$ & 0.7 \\
\hline $6 \mathrm{MWD}[\mathrm{m}]$ & $367.3 \pm 108.1$ & $417.3 \pm 98.9$ & $<0.001$ & $415.4 \pm 71$ & $418.2 \pm 110.7$ & 0.8 \\
\hline Peak $\mathrm{VO}_{2}[\mathrm{ml} / \mathrm{kg} / \mathrm{min}]$ & $16.9 \pm 6.9$ & $16.8 \pm 5.4$ & 0.7 & $13.9 \pm 5.1$ & $18.3 \pm 5$ & 0.02 \\
\hline Peak $\mathrm{VO}_{2}(\%$ of predicted $)$ & $63.7 \pm 23.7$ & $66.5 \pm 20.4$ & 0.6 & $51.5 \pm 14.4$ & $74.8 \pm 18.6$ & $<0.001$ \\
\hline Peak VD/VT & $0.2 \pm 0.08$ & $0.2 \pm 0.07$ & 0.1 & $0.2 \pm 0.05$ & $0.2 \pm 0.08$ & 0.5 \\
\hline VE/VCO 2 slope & $32.5 \pm 8.2$ & $30.9 \pm 6.4$ & 0.1 & $33.7 \pm 7.3$ & $29.3 \pm 5.4$ & 0.046 \\
\hline \multicolumn{7}{|l|}{ Echocardiography: } \\
\hline $\mathrm{EF}(\%)$ & $23.6 \pm 6.1$ & $32.5 \pm 9.5$ & $<0.001$ & $25.5 \pm 8$ & $36.1 \pm 8.1$ & $<0.001$ \\
\hline LVEDV [ml] & $239.2 \pm 77.1$ & $199.3 \pm 92.7$ & 0.06 & $275.6 \pm 113.6$ & $158.3 \pm 41.1$ & $<0.001$ \\
\hline LVESV [ml] & $189.5 \pm 67.6$ & $141.7 \pm 83.8$ & 0.01 & $212.1 \pm 104.2$ & $103.9 \pm 32.6$ & $<0.001$ \\
\hline \multicolumn{7}{|l|}{ Laboratory analyses: } \\
\hline $\mathrm{BNP}[\mathrm{pg} / \mathrm{ml}]$ & $\begin{array}{c}\text { 194, } \\
\text { IQR 71-667 }\end{array}$ & $\begin{array}{c}\text { 166, } \\
\text { IQR 55-521 }\end{array}$ & 0.09 & $\begin{array}{c}\text { 462, } \\
\text { IQR 190-715 }\end{array}$ & $\begin{array}{c}\text { 82.3, } \\
\text { IQR 28-288 }\end{array}$ & 0.01 \\
\hline CRP $[\mathrm{mg} / \mathrm{dl}]$ & $2.7 \pm 3.1$ & $1.9 \pm 1.4$ & 0.1 & $2.2 \pm 1.8$ & $1.75 \pm 1.1$ & 0.3 \\
\hline Erythrocytes $\left[10^{6} / \mu \mathrm{l}\right]$ & $4.4 \pm 0.4$ & $4.47 \pm 0.59$ & 0.5 & $4.5 \pm 0.7$ & $4.4 \pm 0.5$ & 0.8 \\
\hline Hemoglobin [g/dl] & $13.3 \pm 1.3$ & $13.3 \pm 1.7$ & 0.8 & $13.4 \pm 2$ & $13.3 \pm 1.3$ & 0.8 \\
\hline Creatinine $[\mathrm{mg} / \mathrm{dl}]$ & $1.1 \pm 0.3$ & $1.1 \pm 0.4$ & 0.7 & $1.3 \pm 0.4$ & $1 \pm 0.3$ & 0.01 \\
\hline Uric acid [mg/dl] & $6.9 \pm 2.1$ & $7.1 \pm 2$ & 0.6 & $7.8 \pm 2.2$ & $6.6 \pm 1.9$ & 0.1 \\
\hline
\end{tabular}

$6 M$ - heart failure patients after 6 months of cardiac resynchronization therapy, $6 M W D-6$ minute walk test distance, BMI - body mass index, BNP - brain natriuretic peptide, CRP - C-reactive protein, CRT - cardiac resynchronization therapy, EF - ejection fraction, LVEDV left ventricle end-diastolic volume, LVESV - left ventricle end-systolic volume, VDNT - dead space to tidal volume ratio, VE/VCO slope - minute ventilation - carbon dioxide production relationship from the initiation to peak exercise, $\mathrm{VO}_{2}$ - oxygen uptake. 


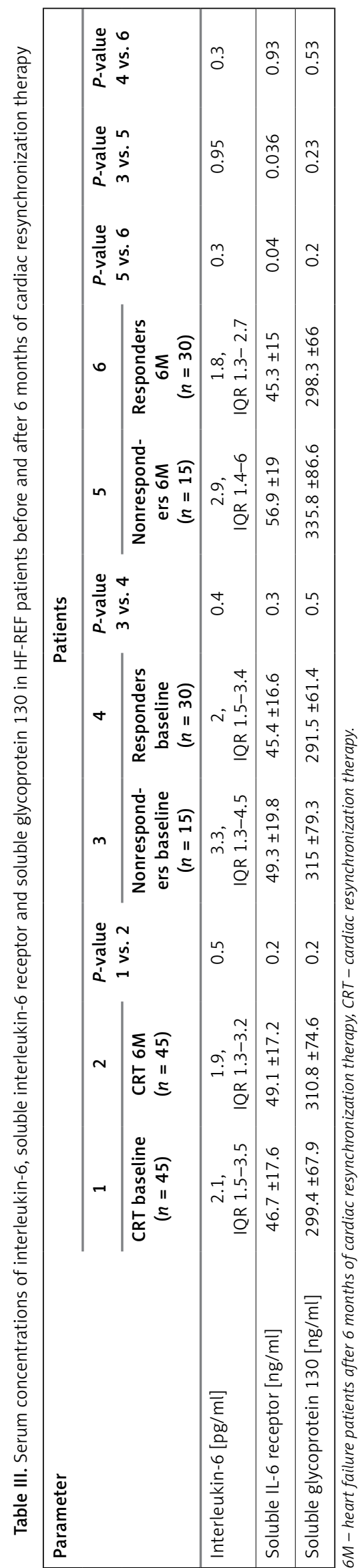

tion did not significantly differ. This observation may generate a hypothesis that sIL-6R decrease is an adaptive anti-inflammatory mechanism in response to chronic IL-6 overexpression in patients with HF-REF. This fact is highlighted by observations on nonresponders, who demonstrated an increase of sIL-6R in the course of CRT.

Another aspect of our study is the link between CRT and changes in the IL-6 signaling. It is known that the increase of heart chambers' load, myocardial injury and underperfusion of peripheral tissues in CHF lead to the increase of cytokine secretion from the mononuclear cells and the myocardium [23]. Previous reports demonstrated that CRT helps to reduce the overload and restore left ventricular systolic function [24]. Based on that knowledge, we hypothesized a CRT-related decrease in pro-inflammatory activation. In our study after 6 months of resynchronization therapy we did not observe a decrease of IL-6 concentration. This observation is probably due to the chronic activation of the inflammatory cascade in heart failure and coexistence of other active chronic cardiovascular comorbidities of known proinflammatory potential, including hypertension or ischemic heart disease. The clinical improvement observed in responders to CRT in our study may not be sufficient to limit IL-6 secretion, especially in the presence of many comorbidities. Moreover, the relatively short follow-up period may not be sufficient to demonstrate either reversal of the inflammatory activation or statistically significant progression, due to the relatively small number of patients who deteriorated. Similar research was conducted by Brouwers, who also did not report differences in markers of inflammation, including IL-6, between responders and nonresponders, while a positive response to CRT was associated with a decrease in BNP and tumor necrosis factor $\alpha$ (TNF- $\alpha$ ) levels [25]. However, the further analysis of HF-REF patients after 6 months of resynchronization therapy showed that those with elevated BNP had higher concentrations of IL-6 and sgp130. This observation as well as Brouwers' findings indicate the fundamental role of BNP in the evaluation of HF-REF patients' status. Another study showed that 48hour CRT interruption resulted in the worsening of left ventricular systolic function, deterioration of mitral regurgitation and increase in serum concentrations of hs-CRP, BNP and IL- 6 in the responders group, while in CRT nonresponders no significant changes were observed [26]. Moreover, this study showed that changes in serum concentrations of IL-6 in responders following CRT interruption correlated with changes in thoracic fluid content (TFC) - a marker of pulmonary congestion [26].

It is still unclear if the increase in IL-6 level is primary or secondary to HF pathogenesis and pro- 
A

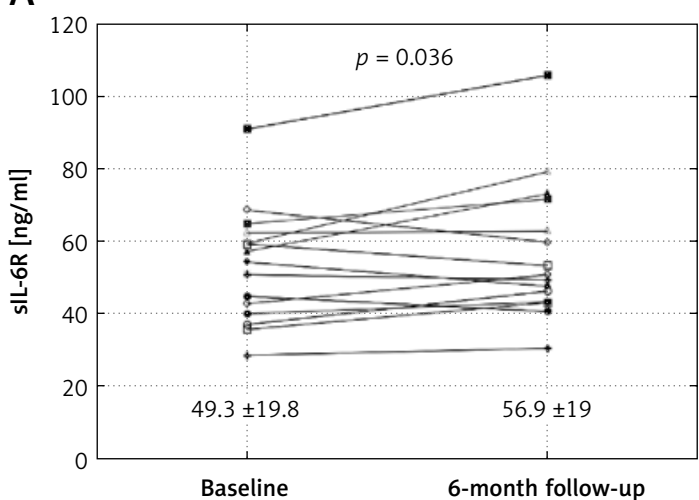

B

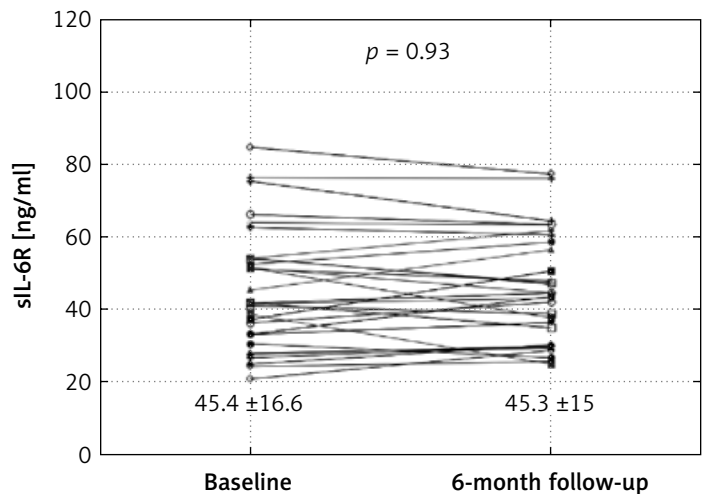

Figure 2. Serum concentrations of soluble interleukin-6 receptor (sIL-6R) in nonresponders (A) and responders (B) before (Baseline) and 6 months after cardiac resynchronization therapy (6-month follow-up). Individual patients are depicted with mean \pm SD given for each group. Difference in time was assessed with the Wilcoxon test

A

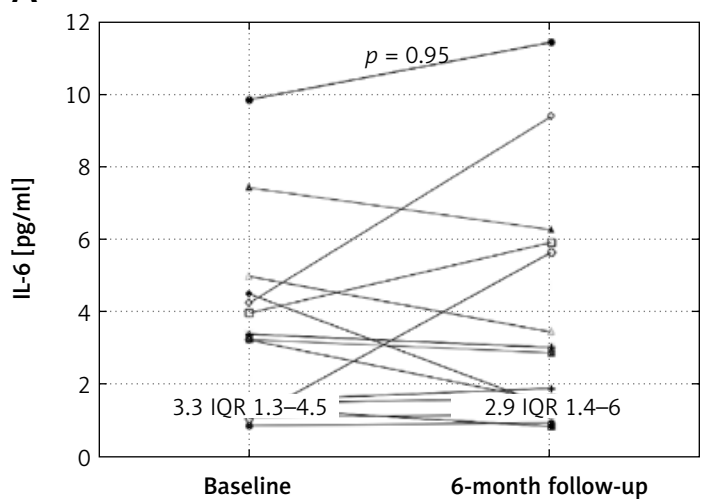

B

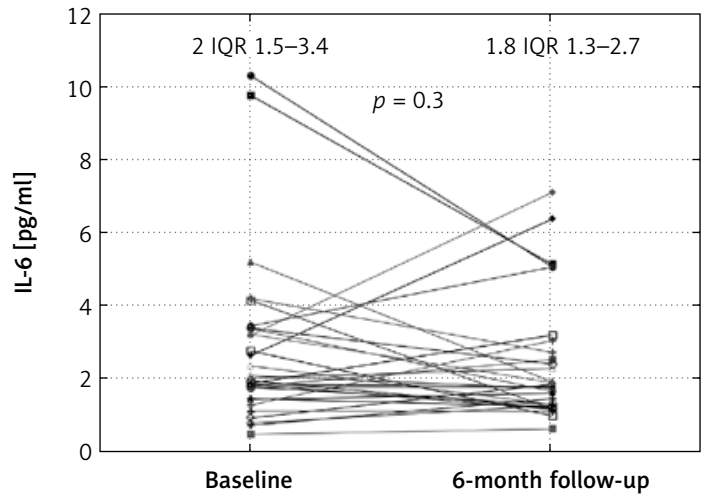

Figure 3. Serum concentrations of interleukin-6 (IL-6) in nonresponders (A) and responders (B) before (Baseline) and 6 months after cardiac resynchronization therapy (6-month follow-up). Individual patients are depicted with median and interquartile range (IQR) given for each group. Difference in time was assessed with the Wilcoxon test

A

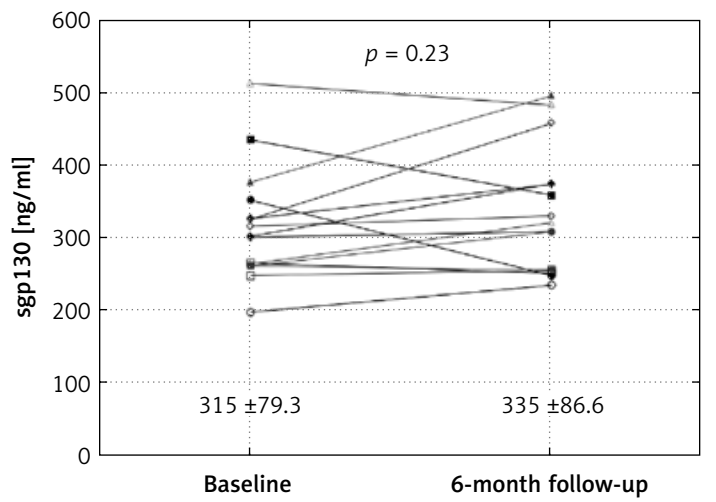

B

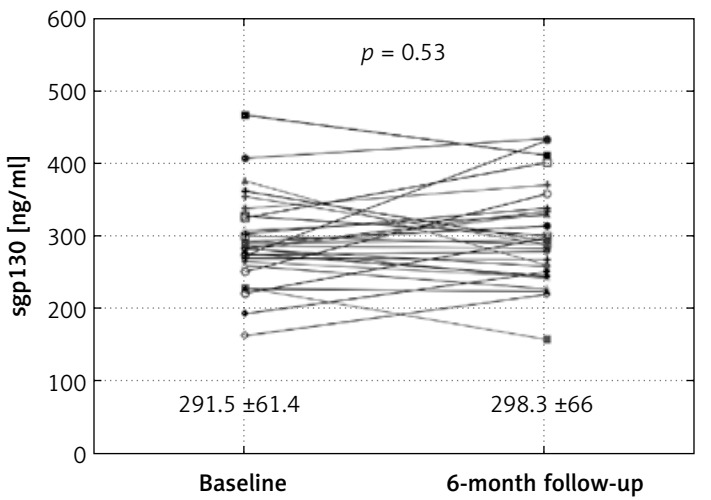

Figure 4. Serum concentrations of soluble gp130 (sgp130) in nonresponders (A) and responders (B) before (Baseline) and 6 months after cardiac resynchronization therapy (6-month follow-up). Individual patients are depicted with mean \pm SD given for each group. Difference in time was assessed with paired Student's $t$ test

gression, while the major role in the disease seems to be pro-inflammatory. This results from the observation that the anti-inflammatory effects of IL-6 are mainly related to classical membrane-bound signaling, while most of the studies underline the pro-inflammatory effect of IL-6 due to trans-signal- ing, with particular contribution to the myocardium [27, 28]. The IL-6 trans-signaling mechanism is largely regulated by local changes in sIL-6R and the ratio of sIL-6R and sgp130 [29]. However, local changes may not reflect the overall mechanisms, which are much more complex. Our data revealed 
the serum alterations in SIL-6R to sgp130 ratio in patients with HF-REF compared to controls. We did not, however, find significant changes of sgp130 in time, which may reflect the more stable part of the IL-6 transduction system, associated with insulin resistance and metabolic disturbances. Our patients represented an optimally treated stable CHF cohort with a relatively short observation period, when few changes occurred with respect to metabolic disturbances. The lack of initial differences between responders and non-responders may be due to the fact that in 6 patients the worse clinical outcome was due to an unsatisfactory percentage of biventricular pacing.

Previous reports on cardiovascular disorders have demonstrated that SIL-6R concentration was elevated in such common conditions as myocardial ischemia and metabolic syndrome and was related to the inflammatory activation of myocardium [30, 31]. In our study basal sIL-6R concentrations correlated with unfavorable parameters of ventilatory inefficiency measured in CPET (VE/VCO ${ }_{2}$ slope). Taken together, these findings highlight that sIL-6R may serve as a switch for IL-6 activation of a maladaptive pathway resulting in disease progression and further HF development. However, there is a lack of data relating to the IL- 6 trans-signaling changes in the context of the CRT response.

In our study, patients who improved after 6 months of CRT had stable sIL-6R levels, while nonresponders showed a trend to increased values. Sgp130 concentrations were comparable between the HF-REF group and controls and remained stable after 6 months of resynchronization therapy. Sgp130 is a product of gp130 transformation, whose expression is relatively stable and less regulated. Increased sgp130 concentration has been associated with many other CV diseases that may cause HF, e.g. ischemic heart disease, metabolic syndrome and hypertension [32, 33]. A large-scale investigation of the associations of sgp130 and IL- 6 with outcomes in ischemic HF (CORONA trial) showed sgp130 to be a strong predictor for overall mortality and CV death, including death from worsening of HF, while IL- 6 did not show an association with any of these [34]. However, in comparison to our study CORONA was a much larger and hence much more heterogeneous population, where the association of sgp130 with metabolic response and insulin sensitivity may play a pathological role [35].

Based on previous reports and our results, we presume trans-signaling to be the main IL- 6 regulatory mechanism that changes in the course of the resynchronization therapy, and this mechanism in large part is based on changes of sIL-6R concentration. These findings indicate that IL-6system changes in the course of $\mathrm{CHF}$ as well as in response to CRT include alterations of both cytokine and soluble receptor levels. Increased sIL-6R concentration seems to be associated with worse clinical status, so its diminished concentration might be considered an adaptive regulatory mechanism decreasing unfavorable IL-6 trans-signaling. This hypothesis based on our results requires further studies. A growing body of evidence suggests that the distinction between the physiological and pathophysiological effects of IL-6 signaling is dependent on the mode of action (trans-signaling vs. canonical pathway).

Our study was based on a relatively small number of selected, optimally treated, stable HF-REF patients, who may not fully represent the whole heterogeneity of the CHF population. However, this selection was vital to investigate potentially small effects that might be lost in a heterogeneous, unselected CHF population.

In conclusion, patients with optimally treated HF-REF present higher IL-6 and lower sIL-6R serum levels than respective controls. CRT-related improvement of the patient's functional status is associated with a smaller change of sIL-6R concentration compared to those who do not benefit from CRT. Thus an unsatisfactory response to CRT is possibly associated with higher IL- 6 activity via trans-signaling. The exact role of the IL- 6 pathway in the CRT response remains to be established.

\section{Acknowledgments}

The first author is the recipient of 'Studiuje, badam, komercjalizuje' grant of the Medical University of Bialystok. The study was supported by statutory grants from the Medical University of Bialystok as well as funds from the Leading National Research Center in Bialystok (KNOW/34/2013 granted to KPK, 153-53815 granted to KK).

The authors are grateful to Maria Alevrontas for linguistic consultation and proofreading.

\section{Conflict of interest}

The authors declare no conflict of interest.

\section{References}

1. Jessup M, Brozena S. Heart failure. N Engl J Med 2003; 348: 2007-18.

2. Straburzyńska-Migaj E, Kałużna-Oleksy M, Maggioni AP, et al. Patients with heart failure and concomitant chronic obstructive pulmonary disease participating in the Heart Failure Pilot Survey (ESC-HF Pilot) - Polish population. Arch Med Sci 2015; 11: 743-50.

3. Abraham WT, Fisher WG, Smith AL, et al. Cardiac resynchronization in chronic heart failure. N Engl I Med 2002; 346: $1845-53$.

4. Auricchio A, Stellbrink C, Butter C, et al. Clinical efficacy of cardiac resynchronization therapy using left ventricular pacing in heart failure patients stratified by severity 
of ventricular conduction delay. J Am Coll Cardiol 2003; 42: 2109-16.

5. Anand IS, Carson P, Galle E, et al. Cardiac resynchronization therapy reduces the risk of hospitalizations in patients with advanced heart failure: results from the Comparison of Medical Therapy, Pacing and Defibrillation in Heart Failure (COMPANION) trial. Circulation 2009; 119: 969-77.

6. Young JB, Abraham WT, Smith AL, et al. Combined cardiac resynchronization and implantable cardioversion defibrillation in advanced chronic heart failure: the MIRACLE ICD Trial. JAMA 2003; 289: 2685-94.

7. Vukajlovic D, Milasinovic G, Angelkov L, et al. Contractile reserve assessed by dobutamine test identifies super-responders to cardiac resynchronization therapy. Arch Med Sci 2014; 10: 684-91.

8. Kusiak A, Wiliński J, Wojciechowska W, et al. Echocardiographic assessment of right ventricular function in responders and non-responders to cardiac resynchronization therapy. Arch Med Sci 2015; 11: 736-42.

9. Hirota $H$, Izumi M, Hamaguchi T, et al. Circulating interleukin- 6 family cytokines and their receptors in patients with congestive heart failure. Heart Vessels 2004; 19: 237-41.

10. Kamiński KA, Oledzka E, Białobrzewska K, Kozuch M, Musiat WJ, Winnicka MM. The effects of moderate physical exercise on cardiac hypertrophy in interleukin 6 deficient mice. Adv Med Sci 2007; 52: 164-8.

11. Chalaris A, Garbers C, Rabe B, Rose-John S, Scheller J. The soluble Interleukin 6 receptor: generation and role in inflammation and cancer. Eur J Cell Biol 2011; 90: 484-94.

12. Narazaki M, Yasukawa K, Saito T, et al. Soluble forms of the interleukin-6 signal-transducing receptor component gp130 in human serum possessing a potential to inhibit signals through membrane-anchored gp130. Blood 1993; 82: 1120-6.

13. Jostock T, Mullberg J, Ozbek S, et al. Soluble gp130 is the natural inhibitor of soluble interleukin- 6 receptor transsignaling responses. Eur J Biochem 2001; 268: 160-7.

14. Plenz G, Song ZF, Tjan TD, et al. Activation of the cardiac interleukin-6 system in advanced heart failure. Eur J Heart Fail 2001; 3: 415-21.

15. Jasiewicz M, Knapp M, Waszkiewicz E, et al. Enhanced IL-6 trans-signaling in pulmonary arterial hypertension and its potential role in disease-related systemic damage. Cytokine 2015; 76: 187-92.

16. Torre-Amione G, Kapadia S, Benedict C, Oral H, Young JB, Mann DL. Proinflammatory cytokine levels in patients with depressed left ventricular ejection fraction: a report from the Studies of Left Ventricular Dysfunction (SOLVD). J Am Coll Cardiol 1996; 27: 1201-6.

17. Eiken HG, Oie E, Damas JK, et al. Myocardial gene expression of leukaemia inhibitory factor, interleukin- 6 and glycoprotein 130 in end-stage human heart failure. Eur J Clin Invest 2001; 31: 389-97.

18. Fischer P, Hilfiker-Kleiner D. Role of gp130-mediated signaling pathways in the heart and its impact on potential therapeutic aspects. Br J Pharmacol 2008; 153 Suppl 1: S414-27.

19. Brignole M, Auricchio A, Baron-Esquivias G, et al. 2013 ESC Guidelines on cardiac pacing and cardiac resynchronization therapy: the Task Force on cardiac pacing and resynchronization therapy of the European Society of Cardiology (ESC). Developed in collaboration with the European Heart Rhythm Association (EHRA). Eur Heart J 2013; 34: 2281-329.
20. Hayes DL, Boehmer JP, Day JD, et al. Cardiac resynchronization therapy and the relationship of percent biventricular pacing to symptoms and survival. Heart Rhythm 2011; 8: 1469-75.

21. Yu CM, Wing-Hong Fung J, Zhang Q, Sanderson JE. Understanding nonresponders of cardiac resynchronization therapy - current and future perspectives. J Cardiovasc Electrophysiol 2005; 16: 1117-24.

22. Chung ES, Leon AR, Tavazzi L, et al. Results of the predictors of response to CRT (PROSPECT) trial. Circulation 2008; 117: 2608-16.

23. Chen Y, Pat B, Gladden J, et al. Dynamic molecular and histopathological changes in extracellular matrix and inflammation in the transition to heart failure in isolated volume overload. Am J Physiol Heart Circ Physiol 2011; 300: H2251-60.

24. Orrego CM, Nasir N, Oliveira GH, et al. Cellular evidence of reverse cardiac remodeling induced by cardiac resynchronization therapy. Congest Heart Fail 2011; 17: 140-6.

25. Brouwers C, Versteeg $\mathrm{H}$, Meine $\mathrm{M}$, et al. Association between brain natriuretic peptide, markers of inflammation and the objective and subjective response to cardiac resynchronization therapy. Brain Behav Immun 2014; 40: 211-8.

26. Rubaj A, Rucinski P, Oleszczak K, et al. Inflammatory activation following interruption of long-term cardiac resynchronization therapy. Heart Vessels 2013; 28: 583-8.

27. Hudzik B, Szkodzinski J, Romanowski W, et al. Serum interleukin- 6 concentration reflects the extent of asymptomatic left ventricular dysfunction and predicts progression to heart failure in patients with stable coronary artery disease. Cytokine 2011; 54: 266-71.

28. Kaminski KA, Kozuch M, Bonda T, et al. Coronary sinus concentrations of interleukin 6 and its soluble receptors are affected by reperfusion and may portend complications in patients with myocardial infarction. Atherosclerosis 2009; 206: 581-7.

29. Rose-John S, Waetzig GH, Scheller J, Grötzinger J, Seegert D. The IL-6/sIL-6R complex as a novel target for therapeutic approaches. Expert Opin Ther Targets 2007; 11: 613-24.

30. Weiss TW, Arnesen H, Seljeflot I. Components of the interleukin- 6 transsignalling system are associated with the metabolic syndrome, endothelial dysfunction and arterial stiffness. Metabolism 2013; 62: 1008-13.

31. Ritschel VN, Seljeflot I, Arnesen H, et al. IL-6 signalling in patients with acute ST-elevation myocardial infarction. Results Immunol 2014; 4: 8-13.

32. Zuliani G, Galvani M, Maggio M, et al. Plasma soluble gp130 levels are increased in older subjects with metabolic syndrome. The role of insulin resistance. Atherosclerosis 2010; 213: 319-24.

33. Inta I, Weber D, Grundt C, et al. Correlation of soluble gp130 serum concentrations with arterial blood pressure. J Hypertens 2009; 27: 527-34.

34. Askevold ET, Nymo S, Ueland T, et al. Soluble glycoprotein 130 predicts fatal outcomes in chronic heart failure: analysis from the Controlled Rosuvastatin Multinational Trial in Heart Failure (CORONA). Circ Heart Fail 2013; 6: 91-8.

35. Nikolajuk A, Kowalska I, Karczewska-Kupczewska M, et al. Serum soluble glycoprotein 130 concentration is inversely related to insulin sensitivity in women with polycystic ovary syndrome. Diabetes 2010; 59: 1026-9. 